\title{
WAIST-TO-HIP RATIO IS THE MOST DISCRIMINATIVE PARAMETER DETECTING OGTT ALTERATIONS IN MEN
}

\author{
Pérez-Pevida $B^{1,2}$, Andrada $P^{1,2}$, Llavero-Valero $M^{1}$, Gutierrez-Buey $\mathbf{G}^{1}$, Núñez-Córdoba $\mathrm{JM}^{3,4,5}$, Gómez-Ambrosi J2,5, Silva \\ $\mathrm{C}^{1,2,5}$, Galofré JC1,5, Salvador $\mathbf{J}^{1,2,5}$, Frühbeck $\mathbf{G}^{1,2,5}$, Escalada $\mathbf{J}^{1,2,5}$. \\ ${ }^{1}$ Department of Endocrinology and Nutrition, Clínica Universidad de Navarra. ${ }^{2}$ Metabolic Research Laboratory. \\ CIBEROBN, Instituto de Salud Carlos III, Clínica Universidad de Navarra. ${ }^{3}$ Division of Biostatistics, Research Support \\ Service, Central Clinical Trials Unit, Clínica Universidad de Navarra. ${ }^{4}$ Department of Preventive Medicine and Public \\ Health, Medical School, Universidad de Navarra. ${ }^{5}$ Navarra Institute for Health Research (IdiSNA). Pamplona, Spain.
}

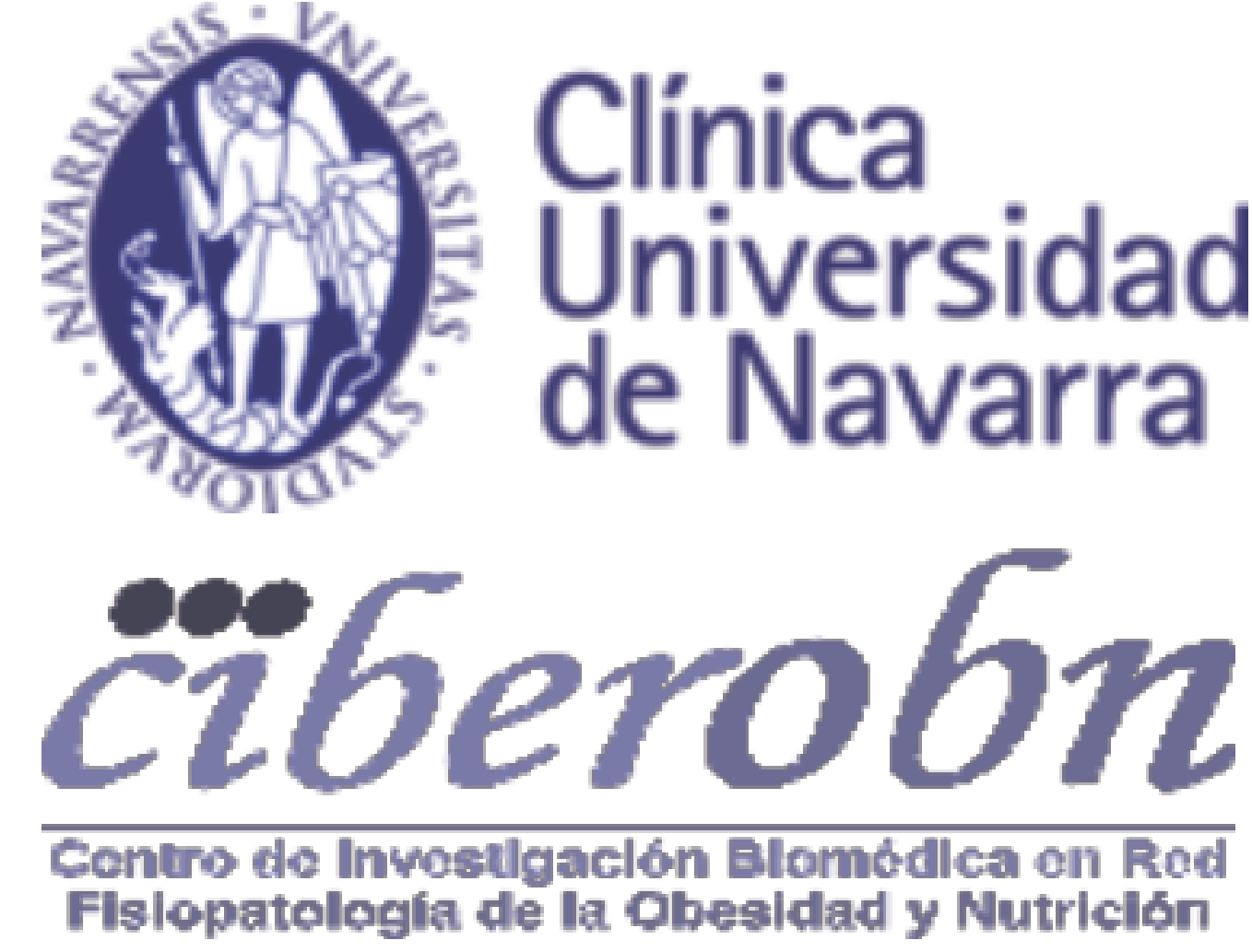

\section{BACKGROUNG AND OBJECTIVES}

\section{Background:}

There is a clear relationship between obesity and carbohydrate metabolism alterations (CMA), such as impaired glucose those alterations, but is not performed in case of normal fasting glucose values (NFG). Moreover, BMI underestimates the diagnosis of obesity and body composition alteration can be crucial to the development of abnormal carbohydrate metabolism. This study aimed to examine the discriminating value of body composition study and anthropometric data compared with $\mathrm{BMI}$ in the diagnosis of CMA.

\section{Objectives:}

- Describe the prevalence of CMA in patients with NFG.

- Study the discriminating value of body composition [total body fat \% (BF\%) and visceral adipose tissue (VAT)] and anthropometric data to detect CMA in men with NFG.

\section{RESULTS}

\section{Variable}

Age, years (mean)

IGT/T2D, \%

Hypertension, \%

Ischemic heart disease, \%

Obstructive sleep apnoea, \%

Smoking, \%

$\mathrm{NASH}, \%$

PAS, $\mathrm{mmHg}$ (mean)

$\mathrm{PAD}, \mathrm{mmHg}$ (mean)

$\mathrm{BMI}, \mathrm{kg} / \mathrm{m} 2$ (mean)

Waist to hip ratio (mean)

Body fat, \% (mean)

Visceral fat, $\mathrm{cm}^{2}$ (mean)

PAL (mean)

Basal insulin, $\mu \mathrm{U} / \mathrm{ml}$ (mean)

HbA1c, \% (mean)

HOMA-IR (mean)

Disposition Index (mean)

Uric acid, mg/dL (mean)

Triglycerides, mg/dL (mean)

LDL-cholesterol, mg/dl (mean)

HDL-cholesterol, mg/dL (mean)

$\mathrm{TSH}, \mu \mathrm{U} / \mathrm{ml}$ (mean)

MDRD, $\mathrm{mL} / \mathrm{min} / 1,73 \mathrm{mE} 2$ (mean)

GPT/GOT index (mean)

Leptin, $\mathrm{ng} / \mathrm{ml}$ (mean)

Fibrinogen, $\mathrm{mg} / \mathrm{dL}$ (mean)

Homocysteine, $\mu \mathrm{mol} / \mathrm{L}$ (mean)
21.6/2.7

24.4

1.7

40.1

26.2

75.64

$127.64 \pm 18.81$

$79.99 \pm 12.42$

$36.05 \pm 8.12$

$1.00 \pm 0.85$

$38,85 \pm 8.39$

$300 \pm 294$

$1.56 \pm 0.12$

$12.92 \pm 8.05$

$5.77 \pm 0.38$

$2.94 \pm 1.87$

$4.22 \pm 3.21$

$6.4 \pm 1.12$

$136 \pm 72.11$

$112.55 \pm 45.52$

$45 \pm 1.34$

$98 \pm 21.53$

$1.47 \pm 0.42$

$30.38 \pm 2.33$

$372,15 \pm 12.06$

$11.66 \pm 0.43$ tolerance (IGT) and T2D. OGTT is used for the diagnosis of

$2.01 \pm 1.10$

\section{METHODS}

We recruited 170 non-diabetic men >18-year-old with NFG ( $\leq 99 \mathrm{mg} / \mathrm{dl})$ who underwent a $75 \mathrm{~g}$ OGTT with a concomitant body composition and anthropometry study between 2000 and 2014.

Body density estimated by Air-Displacement Plethysmography

(Bod-Pod®). BF\% estimated from body density using the Siri equation.

Bioelectrical impedance analysis was used to determine

visceral fat by the ViScan system (Tanita Corp®).

Patients were classified by glucose tolerance on the basis of

blood glucose levels according to WHO criteria for diabetes (2006)

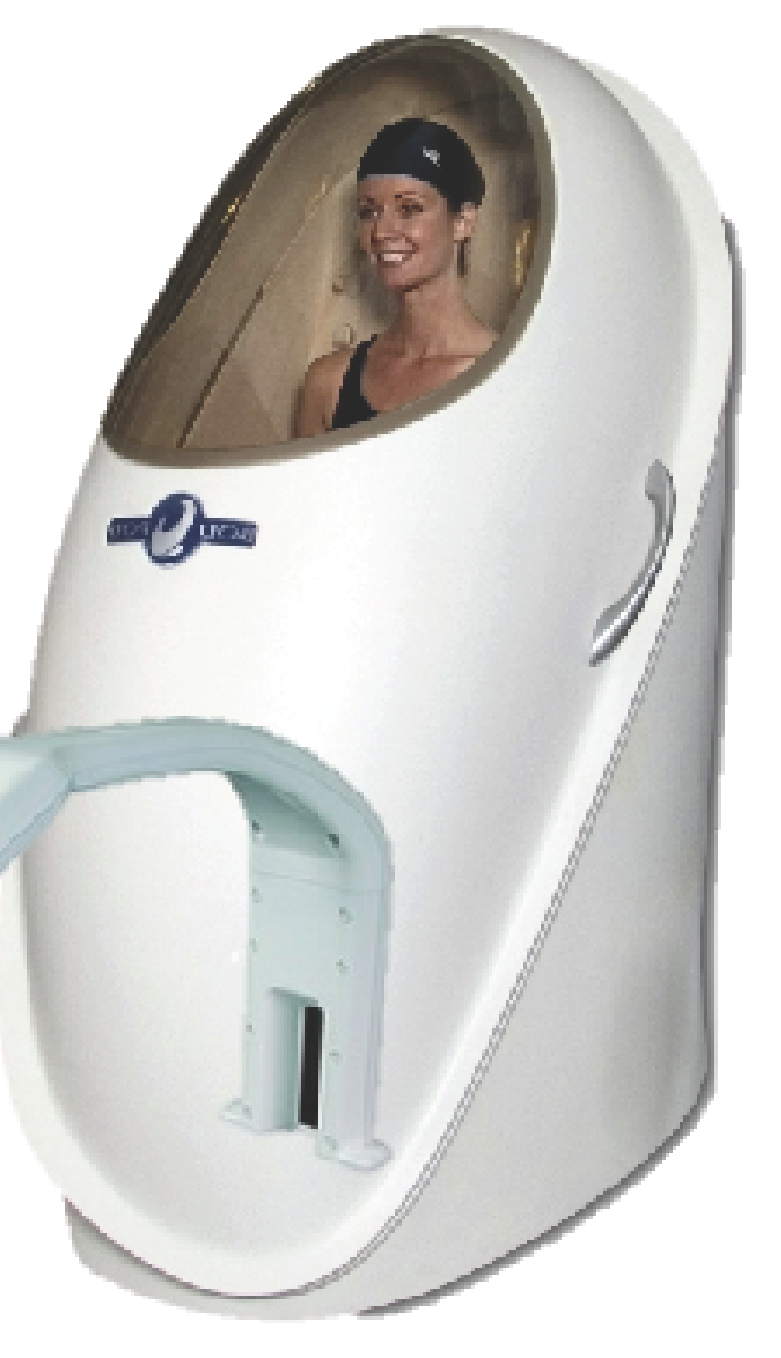

The physical activity level (PAL) was estimated based on a list of the physical activities performed from day to day. Each activity is connected to a number, the physical activity ratio. The PAL was then the time-weighted average of the physical activity ratios

Area Under the ROC Curve (AUC) was used for diagnostic test evaluation.

Figure 1. Discriminative value for detecting CMA of the anthropometric parameters and body composition studies.

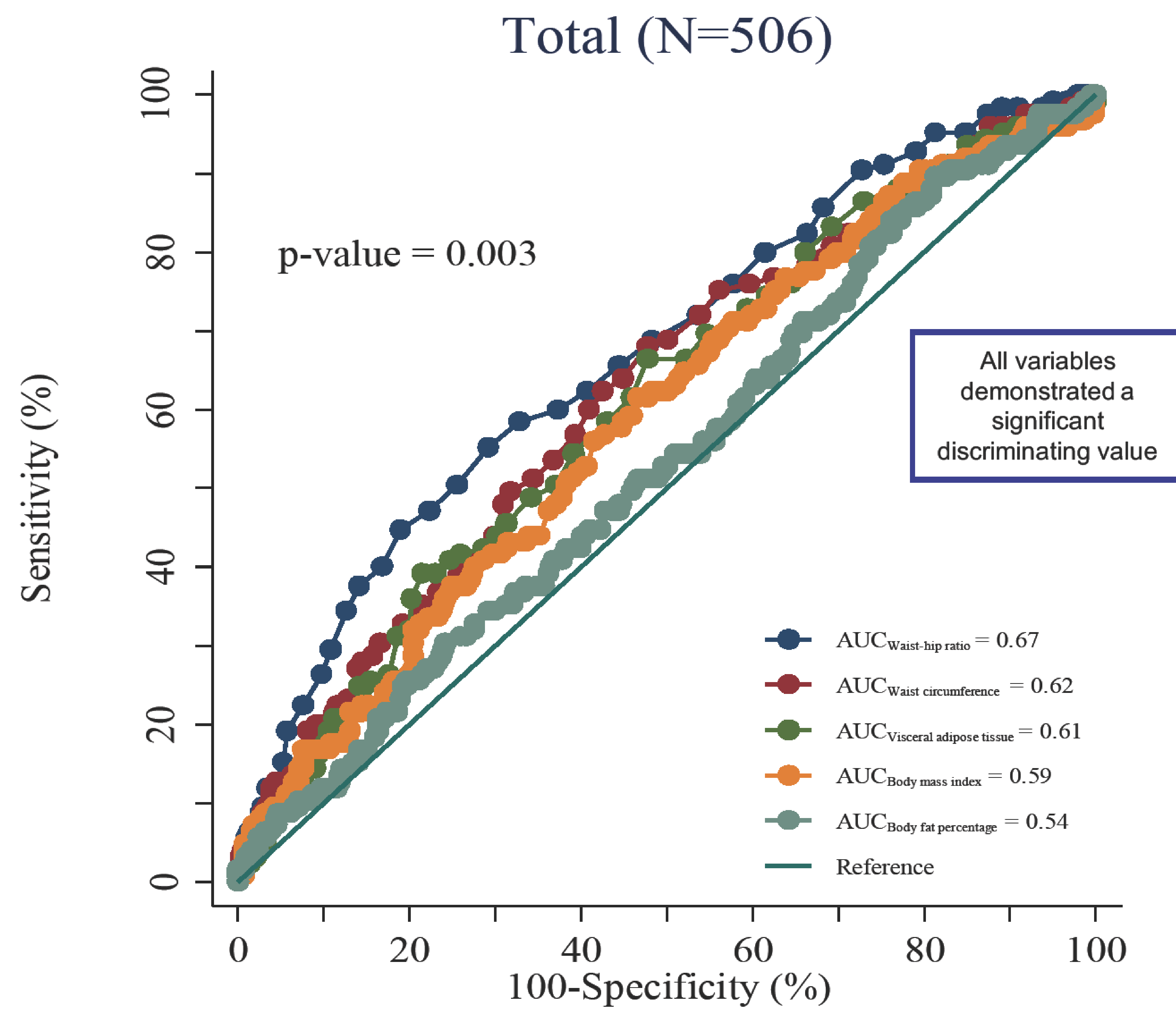

CONCLUSIONS

In our young population, CMA prevalence was $24,3 \%$ after OGTT, so normal fasting glycaemia does not rule out CMA.

Among all the anthropometric and body composition parameters analysed, waist-to-hip ratio was the most discriminative detecting CMA, and it could be helpful to select patients in which an OGTT should be performed.

The early identification of CMA can help to establish appropriate measures to reduce cardiometabolic risk in men.

Keywords: Obesity; Anthropometry; Body composition; Body fat; Visceral fat; Oral glucose tolerance testing.
ECE2016 28 - 31 May 2016 MUNICH, GERM 